\title{
ASYMPTOTIC EXPANSIONS PERTAINING TO THE LOGARITHMIC SERIES AND RELATED TRIGONOMETRIC SUMS
}

\author{
G. FIKIORIS AND P. ANDRIANESIS
}

Abstract. The partial sum of the Maclaurin series of $-\ln (1-z)$ is $f_{n}(z) \equiv \sum_{k=1}^{n-1} z^{k} / k$. We find concise closed-form expressions, involving Eulerian polynomials, for the full asymptotic expansion of $f_{n}(z)$ as $n \rightarrow \infty$. We then use our expressions to find large- $n$ compound asymptotic expansions, involving real quantities only, for $c_{n}(\theta) \equiv \sum_{k=1}^{n-1} \cos k \theta / k, s_{n}(\theta) \equiv \sum_{k=1}^{n-1} \sin k \theta / k$, $r_{n}(\theta) \equiv \sum_{k=0}^{n-1}(-1)^{k} \cos [(2 k+1) \theta] /(2 k+1)$, and a number of other trigonometric sums. Many of these sums are ubiquitous in the literature on the Gibbs phenomenon in the context of Fourier series.

Mathematics subject classification (2010): 33E20, 11L03, 41A60, 11M35.

Keywords and phrases: Logarithmic series, asymptotic expansions, Eulerian polynomials, ApostolBernoulli numbers, trigonometric sums, Lerch's transcendent, Lerch zeta-function.

\section{REFERENCES}

[1] P. Andrianesis And G. Fikioris, Superdirective-type near fields in the Method of Auxiliary Sources, IEEE Trans. Antennas Propag., 60, (2012), 3056-3060.

[2] C. M. BENDER AND S. A. ORSZAG, Advanced mathematical methods for scientists and engineers, McGraw-Hill, §3.7, 1978.

[3] B. C. Berndt, Ramanujan's Notebooks, Part 1, New York, Springer, 1985.

[4] B. C. Berndt, Ramanujan's Notebooks, Part 2, New York, Springer, 1989.

[5] K. N. BoYADZHIEV, A series transformation formula and related polynomials, Int. J. Math. Math. Sci., 23, (2005), 3849-3866.

[6] K. N. BoyadzhIEv, Derivative polynomials for Tanh, Tan, Sech, Sec in explicit form, Fibonacci Quarterly, 45, (2007), 291-303.

[7] K. N. BOYADZHIEv, Apostol-Bernoulli functions, derivative polynomials and Eulerian polynomials, Adv. Appl. Discrete Math., 1, (2008), 109-122.

[8] J. P. BoYD, Acceleration of algebraically-converging Fourier series when the coefficients have series in powers of $1 / n, \mathrm{~J}$. Comput. Phys., 228, (2009), 1404-1411.

[9] T. J. I' A. BRomwich An introduction to the theory of infinite series, 3rd ed. Providence, RI, AMS Chelsea Publishing, p. 324-325, 1991; textually unaltered edition of 2nd ed., London, 1926.

[10] G. Brown And S. Koumandos, A new bound for the Fejér Jackson sum, Acta Math. Hungar., 80, (1998), 21-30.

[11] L. COMTET, Advanced combinatorics: The art of finite and infinite expansions, Dordrecht, Holland, Reidel Publishing Co., 1974.

[12] A. B. O. DAALHUIS, Uniform asymptotic expansions for hypergeometric functions with large parameters I, Anal. Appl., 1, (2003), 111-120.

[13] A. B. O. DAALHUIS, Uniform asymptotic expansions for hypergeometric functions with large parameters II, Anal. Appl., 1, (2003), 121-128.

[14] A. B. O. DAALHUIS, Uniform asymptotic expansions for hypergeometric functions with large parameters III, Anal. Appl., 8, (2010), 199-210.

[15] C. Ferreira AND J. L. LóPeZ, Asymptotic expansions of the Hurwitz-Lerch zeta function, J. Math. Anal. Appl., 298, (2004), 210-224. 
[16] G. Fikioris, I. TASTSOglou And O. N. BAKAS, Selected asymptotic methods with applications to electromagnetics and antennas, Morgan and Claypool Publishers, 2013, Sections 2.4, 4.2.5, and A.2.2.

[17] S. GotTlieb, J.-H. JUng AND S. Kim, A review of David Gottlieb's work on the resolution of the Gibbs phenomenon, Commun. Comput. Phys. 9, (2011), 497-519.

[18] I. S. GRADSHTEYN AND I. M. RYZHIK, Tables of integrals, series, and products, 6th ed. Academic Press, 2000.

[19] R. L. Graham, D. E. Knuth and O. Patashnik, Concrete Mathematics, New York, AddisonWesley, Section 6.2, 1990.

[20] R. W. Hamming, Numerical methods for scientists and engineers, Dover, 1986.

[21] E. Hewitt And R. E. Hewitt, The Gibbs-Wilbraham phenomenon: An episode in Fourier analysis, Arch. Hist. Exact Sci., 21, (1979), 129-160.

[22] A. J. JERRI, The Gibbs phenomenon in Fourier analysis, splines, and wavelet approximations, Kluwer Academic Publishers, Dordrecht, The Netherlands, 1998.

[23] A. J. JERRI, Advances in the Gibbs phenomenon, Sampling Publishing, 2011.

[24] L. B. W. Jolley, Summation of series, Dover Publications, p. 79 and 96, 1961.

[25] S. Kanemitsu, M. Katsurada and M. Yoshimoto, On the Hurwitz-Lerch zeta-function, Aequationes Math., 59, (2000), 1-19.

[26] M. KatsuradA, Power series and asymptotic series associated with the Lerch zeta-function, Proc. Japan Acad., 74, Ser. A, (1998), 167-170.

[27] C. Lanczos, Discourse on Fourier series, Oliver \& Boyd, London, 1966.

[28] Y. L. Luke, The special functions and their approximations, Academic Press Inc., 1969.

[29] Q. M. Luo, On the Apostol-Bernoulli polynomials, Cent. Eur. J. Math., 2, (2004), 509-515.

[30] V. Mangulis, Handbook of series for engineers and scientists, Academic Press, 1965.

[31] T. NAKAmURA, Some formulas related to Hurwitz-Lerch zeta functions, Ramanujan J., 21, (2010), $285-302$.

[32] F. W. J. OLVER, Asymptotics and Special Functions, Originally published New York, Academic Press, 1974; reprinted by AK Peters, Wellesley, MA, 1997.

[33] F. W. J. Olver, D. W. Lozier, R. F. Boisvert and C. W. Clark (Eds.), NiST Handbook of Mathematical Functions, Cambridge University Press, Cambridge, 2010.

[34] A. P. Prudnikov, Y. A. BrychKov AND O. I. Marichev, Integrals and Series, Vol. 1, Elementary Functions. Gordon and Breach, London, U.K., 1986.

[35] Staff of the Bateman Manuscript Project (A. Erdélyi (Editor), W. Magnus, F. Oberhettinger, and F. G. Tricomi (Research Associates)), Higher Transcendental Functions, Vol. I. New York, McGraw-Hill, 1953. (Reprinted, Malabar, FL, Robert Krieger Publishing Co., 1981.)

[36] N. M. Temme, Large parameter cases of the Gauss hypergeometric function, J. Comput. Appl. Math., 153, (2003), 441-462.

[37] E. T. WhitTAKer AND G. N. Watson, A course in modern analysis, 4th ed. Cambridge University Press, 1927; reprinted 2002. p. 151.

[38] R. Wong, Asymptotic Approximations of Integrals, Philadelphia, SIAM, 2001.

[39] A. Zygmund, Trigonometric series, 2nd ed. Cambridge University Press, 1968. 